\title{
Sudden Hearing Loss after Rabies Vaccination
}

\author{
Oğuz Güçlü, Fevzi Sefa Dereköy \\ Department of Otolaryngology, Çanakkale Onsekiz Mart University Faculty of Medicine, Çanakkale, Turkey
}

\begin{abstract}
Background: Sudden hearing loss developing after immunisation is a very rare situation. Rabies is a viral disease characterised by encephalitis and death. Treatment involves active and passive immunisation. Neurologic complications including Guillain-Barre syndrome or facial paralysis are reported in the literature as a side effect after rabies immunisation.

Case Report: Sudden hearing loss was detected in an 11 year-old male patient who had taken the medication for rabies immunisation.

Conclusion: This study presents a case report of sudden hearing loss developing after rabies immunisation - no other aetiological factors were detected and clinical management is discussed in light of the literature.
\end{abstract}

Key Words: Hearing loss, rabies, neurologic, side effect, vaccination, immunisation

Received: 30.12.2012 Accepted: 08.02.2013

\section{Introduction}

The aetiology of sudden hearing loss is multi-factorial and vascular pathologies and viral agents are frequently blamed. Direct invasion of the pathogen, local hypersensitive response to the pathogen or antigen-antibody reactions may cause hearing loss linked to viral agents (1). The result is that patients have sensorioneural hearing loss with or without vertigo and tinnitus. This functional loss may be permanent in some cases, in spite of treatment.

Immunisation is a common method for protecting against viral infections. Side effects developing after immunisation can include sudden hearing loss (SHL), although this is rarely observed. Cases of SHL developing after mumps, measles, hepatitis $B$ and $\mathrm{H} 1 \mathrm{~N} 1$ immunisation are available in the literature (1-4). It is thought that SHL develops due to the immune reaction in these cases. These immunisations generally include the live virus. Similar to how it may develop due to the form of antigen-antibody complex, after immunisation, $\mathrm{SHL}$ may develop due to the triggering of an autoimmune reaction (1).

Neural pathologies (similar to Guillain- Barre, facial paralysis) developing after rabies immunisation are known from the literature (5-9). However, as far as we know, there is no report in the English literature of sudden hearing loss developing after rabies immunisation. This paper will present a case of sudden hearing loss developing after rabies immunisation, and discuss clinical management in light of the literature.

\section{Case Report}

An 11 year old male patient was referred to our clinic with the complaint of hearing loss in his left ear. Hearing loss had begun suddenly on the previous day and was accompanied by rotational vertigo and tinnitus symptoms. The patient had been given the first dose of rabies immunisation due to a bite from a possibly infected dog two days before. Apart from this there was no recent history of upper respiratory infection, medication use, head trauma or systemic illness. Otoscopic examination showed bilateral tympanic membrane and normal middle ear. Weber was lateralised to the left, Rinne was bilaterally positive. Audiological examination indicated right ear $A C / B C$ 82/58 dB, and left ear $A C / B C$ $13 / 10 \mathrm{~dB}$ hearing loss. Sudden hearing loss was diagnosed and the patient was hospitalised. The patient was treated with $0.5 \mathrm{mg} / \mathrm{kg} /$ day methylprednisolone. The patient's haemograms and biochemistry values were within the normal limits. Serological testing showed no anomalies. Magnetic Resonance Imaging of the inner ear with gadolinium was reported to be normal. The patient received the second dose of rabies immunisation whilst treatment continued. On the 5th day of treatment audiological evaluation was performed. Pure tone audiometry thresholds were right ear $\mathrm{AC} /$ BC 30/23 dB and left ear AC/BC 23/17 dB. The patient was discharged under orders to use oral steroids for 5 days more and then report for a check-up. However, the patient did not come to the check-up and applied to our clinic two months later, again with a complaint of hearing loss. It appeared that hearing loss had recurred after the last immunisation dose. Audiometric evaluation indicated hearing thresholds of $A C / B C 93 / 88 \mathrm{~dB}$ in the right ear and 28/27 dB in the left ear. The patient was treated with $1 \mathrm{mg} / \mathrm{kg}$ methylprednisolone for 10 days. Six months after the initial diagnosis, the thresholds determined by audiometry were right ear $A C / B C$ 28/22 dB and left ear 22/15 dB.

Address for Correspondence: Dr. Oğuz Güçlü, Department of Otolaryngology, Çanakkale Onsekiz Mart University Faculty of Medicine, Çanakkale, Turkey. Phone: +902862180393 e-mail: droguzguclu@hotmail.com 


\section{Discussion}

Rabies is a disease characterised by encephalitis and death, developing after a 20-60 day incubation period. In situations with suspicious contact, active (Purified Chick Embryo Cell Vaccine-PCECV or Human Diploid Cell Vaccine-HDCV) or passive (Human Rabies Immune Globulin-HRIG) immunisation is given (8). The aim is to gain enough immune resistance during the incubation period. For active immunisation (PCECV, Rabipur $^{\circledR}$; Novartis vaccines and diagnostics $\mathrm{GmBH}$, Marburg, Germany), our country uses five doses: 0, 3, 7, 14 and 28 days after contact. After the injection, 30-74\% incidence of local (pain, erythema) and $5-40 \%$ incidence of slight systemic (headache, nausea, abdominal pain, dizziness) side effects have been reported $(5,7)$. Neurological complications are very rarely observed. Guillain-Barre syndrome and facial paralysis cases are known from the literature $(5,6)$. The present case described sudden hearing loss developing after rabies immunisation. We did not find any other aetiological factors for sudden hearing loss; it was thought that symptoms could be a complication of the rabies immunisation, but it was also a possible coincidence. The most important point of this case is how sudden hearing loss can be treated in cases following rabies immunisation.

Sudden hearing loss is a rapidly occurring sensory organ function loss. In spite of treatment, in some cases, hearing loss does not resolve. Treatment often involves steroid treatment, peripheral vasodilators, antiviral agents and hyperbaric oxygen treatment. It is thought that sudden hearing loss developing after immunisation may be linked to the immune reaction (1). In these cases, corticosteroids are the general choice for treatment. However, there is insufficient information in the literature about steroid use combined with rabies immunisation. After suspicious contact, immunisation is of vital importance as the disease has a $100 \%$ mortality rate. After immunisation, neutralising antibodies form within 7 days and remain at effective levels for two years. Corticosteroids, immunosuppressive agents and immunosuppressive diseases may change the antibody response. The literature recommends that after immunisation the antibody titre of these patients should be examined to check whether sufficient levels have been reached $(5,8)$. In our case, on the $10^{\text {th }}$ day of the immunisation period, $0.5 \mathrm{mg} /$ $\mathrm{kg} /$ day methylprednisolone was administered. As the patient did not return for check-up after initial treatment, there was no opportunity to check the antibody titre after the immunisation schedule was completed.

In our case, there was a good response to initial treatment but hearing loss then reoccurred. After immunisation, the patient reported that his complaint increased. Two months after the initial application for treatment, this progression of hearing loss responded to $1 \mathrm{mg} / \mathrm{kg} /$ day methylprednisolone treatment. In spite of waiting for two months after the initial occur- rence the situation responded to treatment. In other words steroid treatment may be delayed until after the immunisation period ends. However, this point requires more information and further experiment.

With sudden hearing loss developing after rabies immunisation, another choice that should be considered is intra-tympanic steroid use in place of systemic steroids. This type of administration may be an advantage as it does not systemically suppress the immune system.

\section{Ethics Committee Approval: N/A.}

Informed Consent: Informed consent was obtained from parents of the participant.

Peer-review: Externally peer-reviewed.

Author contributions: Concept - O.G., F.S.D.; Design - O.G., F.S.D.; Supervision - O.G., F.S.D.; Resource - O.G., F.S.D.; Materials - O.G., F.S.D.; Data Collection\&/or Processing - O.G., F.S.D.; Analysis\&/or Interpretation - O.G., F.S.D.; Literature Search - O.G., F.S.D.; Writing O.G., F.S.D.; Critical Reviews - O.G., F.S.D.

Conflict of Interest: No conflict of interest was declared by the authors.

Financial Disclosure: No financial disclosure was declared by the authors.

\section{References}

1. Huang $\mathrm{HH}$, Huang CC, Hsueh PY, Lee TJ. Bilateral sudden deafness following H1N1 vaccination. Otolaryngol Head Neck Surg 2010:143;849-50. [CrossRef]

2. Kaga K, Ichimura K, Ihara M. Unilateral total loss of auditory and vestibular function as a complication of mumps vaccination. Int $J$ Pediatr Otorhinolaryngol 1998:43;73-5. [CrossRef]

3. Orlando MP, Masieri S, Pascarella MA, Ciofalo A, Filiaci F. Sudden Hearing Loss in Childhood Consequent to Hepatitis B Vaccination. Ann N Y Acad Sci 1997:830;319-21. [CrossRef]

4. Asatryana A, Poolb V, Chenb RT, Kohlb KS, Davis RL, Iskanderb JK. Live attenuated measles and mumps viral strain-containing vaccines and hearing loss: Vaccine Adverse Event Reporting System (VAERS), United States, 1990-2003. Vaccine 2008;26:1166-72. [CrossRef]

5. National Advisory Committee on Immunization (2006) Canadian Immunization Guide, Seventh Edition, Minister of Supply and Services, Canada

6. Crémieux G, Dor JF, Mongin M. Peripheral facial paralysis and post-antirabies-vaccination polyneuroradiculitis. Acta Neurol Belg 1978:78;279-300.

7. Mattner F, Bitz F, Goedecke M, Viertel A, Kuhn S, Gastmeier P, et al. Adverse effects of rabies preand postexposure prophylaxis in 290 Health-care-workers exposed to a rabies infected organ donor or transplant recipients. Infection 2007;35:219-24. [CrossRef]

8. Hendekli CM. Current therapies in rabies. Arch Virol 2005:150;1047-57. [CrossRef]

9. Dobardzic A, Izurieta H, Woo EJ, Iskander J, Shadomy S, Rupprecht $C$, et al. Safety review of the purified chick embryo cell rabies vaccine: Data from the Vaccine Adverse Event Reporting System (VAERS), 1997-2005. Vaccine 2007;25:4244-51. [CrossRef] 